\section{Fehler in der}

\section{Pharmakotherapie und mögliche Vorbeugungs- massnahmen*}

\author{
M. Giger, B. Hardmeier ${ }^{a}$, K. Hartmann ${ }^{b}$, K. Fattinger ${ }^{a}$, M. Kuhn ${ }^{b}$, \\ P. Meier-Abt ${ }^{a}$ \\ a Stiftung für Arzneimittelsicherheit/CHDM \\ b Schweizerische Arzneimittel-Nebenwirkungs-Zentrale
}

Fehler in der Pharmakotherapie sind das Resultat falschen Rezeptierens bzw. falscher Applikation von Arzneimitteln. Ursächlich handelt es sich am häufigsten um missachtete Allergien und Interaktionen sowie Dosierungsfehler. Fehler in der Pharmakotherapie sind für beinahe 1 Prozent der Hospitalisationen verantwortlich. Durch Intensivierung der Aus-, Weiter- und Fortbildung, einen rationalen Arzneimitteleinsatz, einen offenen Umgang mit Fehlern sowie ein elektronisch unterstütztes Rezeptieren mittels Decision Support Systems (DSS) kann weiteren Fehlern vorgebeugt werden.

Die Pharmakotherapie wird sehr breit angewendet. Zwei von drei ambulanten Konsultationen enden mit der Einleitung oder Fortsetzung einer medikamentösen Therapie. Dabei werden immer wirksamere Arzneimittel eingesetzt. Zu den umsatzstärksten Arzneimitteln zählen die nichtsteroidalen Antirheumatika (NSAR). Diese sind aufgrund ihres Nebenwirkungspotentials für rund $2 \%$ der internmedizinischen Hospitalisationen verantwortlich. Oft werden diese Wirkstoffe mit falscher Indikation oder trotz Kontraindikation eingesetzt. Dieses Beispiel wirft die Frage einerseits nach dem sicheren Umgang mit Arzneimitteln und andererseits nach dem korrekten Einsatz von Arzneimitteln auf.

Die Arzneimittelsicherheit wird durch die Registrierungsbehörde mittels eines Spontanmeldesystems

\footnotetext{
* In Anlehnung an das gleichnamige Referat anlässlich der FMH-Tagung "Risiken, Fehler und Patientensicherheit», Bern, 25. Januar 2001.
}

Korrespondenz:

Dr. med. Max Giger

Mitglied Zentralvorstand FMH

Oberer Deutweg 59

CH-8400 Winterthur überwacht. So werden unerwünschte Arzneimittelwirkungen, die während der klinischen Testung nicht erfasst wurden, erkannt. In der Schweiz werden seit zwanzig Jahren durch die Ende 1979 von der Verbindung der Schweizer Ärztinnen und Ärzte/FMH und der Schweizerischen Gesellschaft für Chemische Industrie (SGCI) gegründete Schweizerische Arzneimittel-Nebenwirkungs-Zentrale (SANZ) auf privater Basis freiwillig gemeldete, vermutete unerwünschte Arzneimittelwirkungen (UAW) gesammelt, ausgewertet und an die Registrierungsbehörde (IKS) gemeldet. Die SANZ muss leider, bedingt durch die Rahmenbedingungen des Heilmittelinstitutes, welches nach Inkrafttreten des Heilmittelgesetzes die IKS ablöst, ihre erfolgreiche Tätigkeit Mitte 2001 einstellen. Die Institute für klinische Pharmakologie und Toxikologie der fünf Universitäten sammeln auch UAW und melden diese der IKS. Prospektiv und komprehensiv werden Arzneimittelwirkungen im Rahmen des EventMonitoring der Stiftung für Arzneimittelsicherheit (CHDM) an der medizinischen Klinik des Universitätsspitals Zürich und des Kantonsspitals St. Gallen gesammelt und ausgewertet. Die Resultate dieser Untersuchungen erlauben folgende Aussagen bezüglich der Arzneimittelsicherheit in der Schweiz: 3,0\% der Hospitalisationen auf internmedizinischen Kliniken erfolgten aufgrund von UAW; bei 10\% der hospitalisierten internmedizinischen Patienten traten während des Spitalaufenthaltes klinisch relevante UAW auf. In 0,15\% aller Hospitalisationen waren UAW für Todesfälle verantwortlich $[1,2]$. Diese Zahlen lassen noch keine Rückschlüsse auf allfällige Fehler zu.

Der Begriff «Fehler» bzw. Fehlverhalten muss vorgängig der Beurteilung einer Sachlage, im vorliegenden Falle eines "Therapiefehlers», definiert werden. Im nachhinein können «Fehler» nur noch nach sogenannt allgemein gültigen Standards ("State of the art») beurteilt werden. Es fehlen dann oft zusätzliche Informationen zum Prozess der Arzneimittelverordnung und Arzneimittelapplikation, in welchem die eigentlichen Fehler geschehen, die zum unerwünschten Resultat, d.h. der nichtbeabsichtigten unerwünschten Arzneimittelwirkung, führen. Ein grosses Problem besteht in der Pharmakotherapie von Kindern und Jugendlichen infolge des weitverbreiteten Einsatzes von Arzneimitteln ausserhalb der von der Arzneimittelbehörde registrierten Altersgruppe ("off label use») und infolge der galenischen Veränderung einer Arzneimittelspezialität für den Einsatz in der Pädiatrie ("unlicensed use») [3, 4]. Hier von Fehlern zu sprechen, wäre falsch; Abhilfe können einzig prospektive Studien über die pädiatrische Pharmakotherapie, und zwar schon vor der Registrierung eines neuen Arzneimittels, schaffen. Die Definition der ärztlichen Fehler ist nur ein Teil eines grossen Prozesses, in welchem es um die Verhütung von Fehlverhalten durch Eingestehen von Fehlern und deren Untersuchung geht [5-7]. Die Fehler im Rahmen der Pharmakotherapie können wie in Tabelle 1 eingeteilt werden: 
Tabelle 1

Fehler in der Pharmakotherapie.

Produktfehler
- Falscher Inhalt
- Verfalldatum überschritten
- Verunreinigung
Ärztliche Fehler
- Falsche Indikation (Diagnose und Arzneimittel passen nicht
aufeinander, u. a. Verwechslung)
- Fehlende Indikation (Falscher Einsatz des Arzneimittels)
- Verpassen von Kontraindikationen
- Missachten potentieller Interaktionen
- Absolut falsche Dosierung
- Relativ falsche Dosierung (Grundleiden)
- Falsche Applikation (örtlich/zeitlich)
- Inadäquate Überwachung
Patientenfehler
- Selbstmedikation mit falschem Arzneimittel
- Selbstmedikation mit falscher Dosierung
- Nichtbefolgen ärztlicher Anweisungen

Produktfehler können schwere Folgen zeitigen; ihre Verhinderung sollte durch die Qualitätsanforderungen an den Herstellungsprozess und die Lagerhaltung erfolgen. Oft werden auch eindeutige Produktfehler als "medizinische oder ärztliche Fehler» apostrophiert. So handelte es sich bei der Anfang 2001 am Berner Inselspital aufgetretene "Verwechslung" von Glukose und Kalziumchlorid um einen auf den Herstellungsprozess zurückzuführenden Produktfehler. Patientenfehler sind bei der Beurteilung eines unerwarteten Ereignisses unter Pharmakotherapie immer in Betracht zu ziehen. Die sogenannte Selbstmedikation, meistens mit freiverkäuflichen (sogenannte OTC-Produkte) häufig auch mit der Rezeptpflicht unterstellten Produkten, die von früheren oder fremden Behandlungen stammen, wird von den Patienten oft unbewusst verschwiegen. Weit verbreitet ist der unkontrollierte Konsum von Tranquilizern und NSAR. Die Problematik der ungenügenden Compliance oder falschen Einnahme von Arzneimitteln, die zum Teil kommunikationsbedingt sind, sei nur kurz erwähnt. An dieser Stelle sollen Patientenfehler einzig erwähnt und lediglich auf die ärztlichen Fehler näher eingetreten werden.

\section{Fehler in der Pharmakotherapie: Schweizer Daten}

Die ärztlichen Fehler werden bis heute in der Schweiz nicht systematisch erfasst. Auch im bewährten Spontanmeldesystem der SANZ erfolgte bei der Beurteilung von vermuteten unerwünschten Arzneimittelwirkungen keine systematische Registrierung von Pharmakotherapiefehlern, da deren Definition nicht abschliessend erfolgen kann. Da bei der Bearbeitung der eingegangenen Meldungen den als schwerwiegend beurteilten Zwischenfällen vermehrte Aufmerk- samkeit zukam, wurden einzig in diesen Fällen vorliegende sogenannte "Medikationsfehler" registriert. So darf aus den folgenden Daten nicht auf die Inzidenz von Medikationsfehlern bei gemeldeten unerwünschten Arzneimittelwirkungen geschlossen werden. Unter den über 17000 Meldungen in der SANZDatenbank waren bei 32 Meldungen aufgrund des allgemein anerkannten Kenntnisstandes über den korrekten Arzneimitteleinsatz ärztliche Fehler Grund für die oft schwere, in einem Fall tödlich verlaufene Arzneimittelreaktion (Tab. 2).

In dieser Aufstellung sind zwei Meldungen über Darmperforation nach fehlerhafter Applikation eines Einlaufs, über eine Peritonitis nach Pertubation wegen falscher bzw. zu tiefer Applikation einer Vaginalspülung sowie über 4 Meldungen von Luftembolien nach Verabreichung einer kolloidalen Infusionslösung (Polygeline) nicht enthalten.

Die Datenbank der Stiftung für Arzneimittelsicherheit SAS/CHDM, welche prospektiv und komprehensiv alle Patienten bezüglich Arzneimitteltherapie und die damit in Zusammenhang stehenden Wirkungen sowie deren Vital- und Labordaten an definierten Abteilungen der Kliniken für Innere Medizin am Universitätsspital Zürich und am Kantonsspital St. Gallen erfasst, enthält keine Einschätzung über die korrekte Durchführung der Pharmakotherapie. So konnten Therapiefehler vorläufig einzig im nachhinein aufgrund des "State of the art" des korrekten Arzneimitteleinsatzes bei Patientengruppen mit arzneimittelbedingten Reaktionen erfasst werden. Die Daten derjenigen 183 Patienten, bei welchen eine Hospitalisation infolge der Arzneimitteltherapie (3\%) erfolgte, wurden bezüglich Fehler in der Arzneimitteltherapie untersucht. Dabei stellte sich heraus, dass 48 von 6133 Hospitalisationen fehlerbedingt waren: 12 mal lagen Patientenfehler (0,2\%) vor, unter anderem traten 4 Gastrointestinalblutungen nach nichtindizierter Einnahme von NSAR auf. Bei 38 medikamentenbedingten Hospitalisationen lagen ärztliche Fehler (0,6\%) (Tab. 3) vor; zweimal führten sie zum Tod $(0,03 \%)$.

Auch diese Daten erlauben keine Rückschlüsse auf die Inzidenz von ärztlichen Fehlern in der Pharmakotherapie in der Ambulanz; wurden doch einzig schwerwiegende Fehler, nämlich solche, welche zur Hospitalisation führten, erfasst. Die effektive Fehlerquote dürfte höher liegen. Eine Auswertung der prospektiv erfassten Therapiedaten während der Hospitalisation wird die Inzidenz von Fehlern in der Pharmakotherapie, wohlgemerkt im nachhinein definiert, auf internmedizinischen Kliniken aufzeigen können.

\section{Fehler in der Pharmakotherapie: Literaturdaten}

Systematische Datensammlungen zu Fehlern in der Pharmakotherapie finden sich in der Literatur nur vereinzelt. Hier soll einerseits auf die systematische Kontrolle der ärztlichen Rezepte in der Spitalapotheke und andererseits auf das elektronisch unterstützte Rezeptieren näher eingegangen werden. 
Tabelle 2

Ärztliche Fehler (SANZ-Daten).

\begin{tabular}{lll}
\hline Falsche Indikation & 4 & Medikamentenverwechslungen \\
\hline Verpassen von Kontraindikationen & 14 & $\begin{array}{l}\text { 12 Ketokonazol bei Nagelmykose, } \\
\text { 2 i.v. Applikation bei bekannter Allergie } \\
\text { (Diclofenac; Metamizol mit Exitus letalis) }\end{array}$ \\
\hline Falsche Dosierung & 3 & Loperamid, Disulfiram, Tropanalkaloid \\
\hline Falsche Applikation & 11 & $\begin{array}{l}\text { 5 intrathekale anstatt intravaskuläre } \\
\text { Applikationen (Röntgenkontrastmittel, } \\
\text { Erythromycin), 2 i.v. anstatt i.m. (Eisen- } \\
\text { dextran, Depot-Penicillin), 1 i.v. anstatt } \\
\text { p.o. (Röntgenkontrastmittel), i.v. anstatt } \\
\text { lokal (Antiseptikum), i.m. bei M. Osler }\end{array}$ \\
\hline
\end{tabular}

\section{Tabelle 3}

Ärztliche Fehler (SAS/CHDM-Daten).

\begin{tabular}{|c|c|c|}
\hline Falsche Indikation & 1 & Aredia bei Thoraxschmerzen \\
\hline Fehlende Indikation & 7 & NSAR als Analgetika, Antikoagulation \\
\hline Verpassen von Kontraindikationen & 2 & $\begin{array}{l}\text { Antikoagulation, NSAR bei Nieren- } \\
\text { insuffizienz }\end{array}$ \\
\hline Missachten potentieller Interaktionen & 13 & $\begin{array}{l}\text { Antikoagulation und NSAR, Rifampicin } \\
\text { und Ciclosporin, Betablocker und } \\
\text { ACE-Hemmer, Diuretika, Isoptin und } \\
\text { Cordarone, Azathioprin und Allopurinol }\end{array}$ \\
\hline Absolut falsche Dosierung & 3 & Insulin, Nivaquin, Tacrolimus \\
\hline Relativ falsche Dosierung & 3 & $\begin{array}{l}\text { Digoxin bei Niereninsuffizienz, } \\
\text { Insulin bei Inappetenz, Übersedierung } \\
\text { (Tranquilizer, Neuroleptikum) }\end{array}$ \\
\hline Falsche Applikation & 2 & $\begin{array}{l}\text { Methotrexat (1 Exitus letalis infolge } \\
\text { aplast. Anämie) }\end{array}$ \\
\hline Inadäquate Überwachung & 7 & $\begin{array}{l}\text { Antikoagulation, ACE-Hemmer } \\
\text { (Exitus letalis infolge Angioödem) }\end{array}$ \\
\hline
\end{tabular}

\section{Rezeptkontrolle [8]}

Die Kontrolle der Rezepte in der Spitalapotheke eines 631-Betten-Spitals ergab zwischen 4,1 und 8,5 Fehler pro 1000 ausgestellte Rezepte für die medizinische bzw. chirurgische Abteilung. 6,2\% der Fehler wurden als potentiell schwer bis tödlich und 13,8\% als potentiell schwerwiegend eingestuft. Ursächlich lagen den Fehlern vor allem eine Fehlinterpretation der Krankheit (13,9\%), Missachtung von bekannten Allergien (12,1\%), falsche Dosierung (11,1\%) oder ein falsches Dosisintervall (10,8\%) zugrunde. An der Spitze der Fehlertypen lagen Überdosierungen $(41,8 \%)$, gefolgt von Unterdosierungen $(16,5 \%)$, missachteten Allergien (12,9\%) und falschen Applikationen $(11,6 \%)$.

\section{EDV-unterstütztes Rezeptieren [9]}

Während 21 Monaten erfolgte an einer 64-BettenNephrologie mit je 500 Patienten unter Hämodialyse bzw. nach Nierentransplantation das Ausstellen von Rezepten mit Hilfe eines sogenannten «Decision Sup- port Systems" (DSS). Dabei erfolgte nach Eingabe der Patienten- und Arzneimitteldaten bei korrektem Verschreiben keine Meldung, bei fehlerhaftem Verschreiben drei Arten von Meldungen, je nach möglicher Folge der Fehlverschreibung ein Verbot (0,07\%), eine Warnung $(0,48 \%)$ oder ein Hinweis $(1,43 \%)$. Aufgrund dieses DSS wurden 2\% der 87789 für 1646 Patienten ausgestellten Rezepte nicht ausgeführt. Es handelte sich ursächlich um Kontraindikationen infolge nichtbeachteter Allergie, potentielle Interaktionen und Dosierungsfehler.

\section{Umgang mit Fehlern und mögliche Vorbeugungs- massnahmen}

Fehler in der Pharmakotherapie sind das Resultat eines komplexen interaktiven Prozesses. Als solche müssen sie systematisch und prozessorientiert erfasst werden. Dies kann durch das komprehensive prospektive Eventmonitoring während der Pharmakotherapie an stationären und ambulanten Patienten erfolgen. Dazu werden allgemeine Patientendaten, anamnestisch relevante Daten (Allergien!), Vitaldaten, Labordaten und Arzneimitteldaten benötigt. Die Fehler müssen analysiert werden. Dies setzt voraus, dass die Fehler als solche erfasst werden und deren Existenz bewusst gemacht und kommuniziert wird. So können im Rahmen des Qualitätssicherungsprozesses Massnahmen zur Verhinderung initiiert werden (Abbildung 1).

Folgende Massnahmen können zur Sicherung der Pharmakotherapie und zur Fehlerprävention eingesetzt werden:

Steigerung der Strukturqualität durch Aus-,

Weiter- und Fortbildung

- Einbau der Pharmakotherapie in alle klinischen Weiterbildungsprogramme;

- Systematisch supervisioniertes Rezeptieren an den Weiterbildungsstätten;

- Qualitätszirkel;

- Guidelines.

\section{Steigerung der Prozessqualität}

- Systematisches Rezeptieren mit dem Ziel eines rationalen Arzneimitteleinsatzes;

- Rezeptieren unter Zuhilfenahme von DSS;

- Elektronisch unterstützte/gesteuerte Abgabe der Arzneimittel;

- Eventmonitoring.

Die Massnahmen zur Steigerung der Strukturqualität werden im Rahmen der Umsetzung der neuen Weiterbildungsordnung (WBO) mittels schriftlicher Weiterbildungskonzepte für alle Weiterbildungsstätten und den geplanten Visitationen derselben sowie des Ausbaus der Fortbildung im Sinne einer beruflichen Fitness bzw. des Continuing professional development (CPD) eingeleitet. Diese Massnahmen überschneiden sich zum Teil mit denjenigen zur Steigerung der Prozessqualität. Heute bestehen zwar gut ausgebaute 


\section{Abbildung 1}

Umgang mit Fehlern.

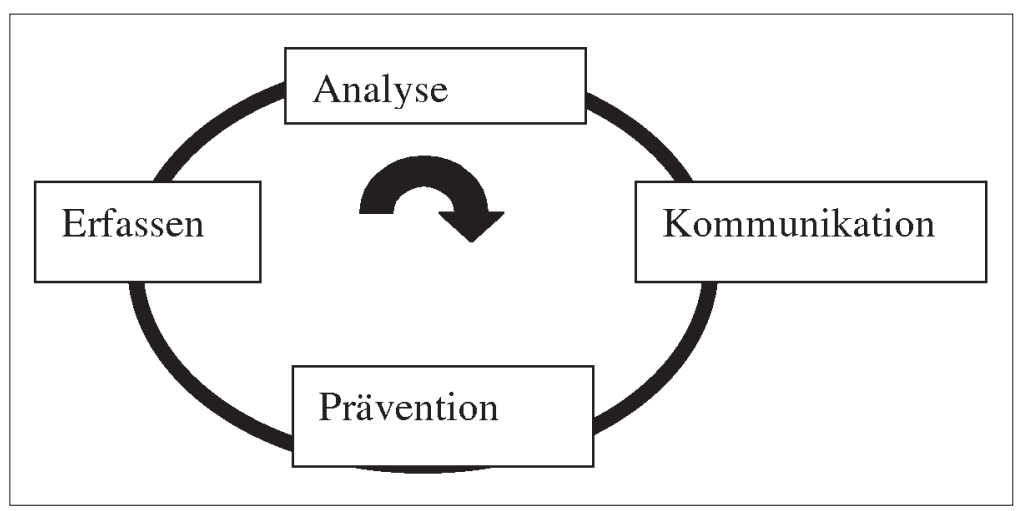

Arzneimitteldatenbanken mit Angaben zu allen pharmakotherapeutisch relevanten Fragen wie Wirkungen, Nebenwirkungen und Interaktionen sowie Dosierung und Applikationswege, die zur Unterstützung des Rezeptierens herangezogen werden können [10]. Solche übers Internet abrufbare Ressourcen werden jedoch nur von 7\% der Schweizer Grundversorger trotz weitverbreiteter Internetzugänglichkeit im praktischen Alltag bzw. bei therapeutischen Entscheidungen während der Sprechstunde eingesetzt [11]. Dies ist darauf zurückzuführen, dass sie noch nicht in einer benutzerfreundlichen Art, durch einfache Stichworteingabe einerseits und auf derselben elektronischen Ebene andererseits vorliegen wie die notwendigen Patientendaten. Decision Support Systems (DSS), welche online einen praktisch gleichzeitigen, d.h. bei vorliegenden Patientendaten, einfachen Zugriff auf solche Datenbanken erlauben, werden entwickelt. Ihnen gehört die Zukunft im Interesse einer prozessorientierten sichereren Pharmakotherapie. Dem Rezeptieren mit Unterstützung des DSS folgt die Abgabe der Arzneimittel an Patientinnen und
Patienten. In der Ambulanz können bis zur Auslieferung an die Abgabestelle, im Spital bis zur Administration [12] durch elektronische Übermittlung des Rezeptes und robotergesteuertes Dispensieren weitere Fehler verhindert werden. Das Eventmonitoring ermöglicht einerseits Wirkungen und andererseits UAW bzw. mögliche Fehler zu erfassen, aus welchen Schlüsse zur weiteren Prozessoptimierung gezogen werden können.

\section{Literatur}

1 Fattinger KE, Roos M, Vergères P, Holenstein C, Kind B, Masche U, et al. Epidemiology of drug exposure and adverse drug reactions in two Swiss departments of internal medicine. Br J Clin Pharmacol 2000;49:158-67.

2 Fattinger KE, Roos M, Spiess A, Vergères P, Braunschweig S, Kullak-Ublick GA, et al. Die «Stiftung für Arzneimittelsicherheit» SAS/CHDM: Update 2000. Schweiz Ärztezeitung 2000;81:2381-4.

3 Turner S, Nunn AJ, Fielding K, Choonara I. Adverse drug reactions to unlicensed and off-label drugs on paediatric wards: a prospective study. Acta Paediatr 1999;88:965-8.

4 Conroy S, Choonara I, Impicciatore P, Mohn A, Arnell H, Rane A, et al. Survey on unlicensed and off label drug use in paediatric wards in European countries. Br Med J 2000; 320:79-82.

5 Brennan TA. The institute of medicine report on medical errors - could it do harm? N Engl J Med 2000;342:1123-5.

6 Weingart SN, Wilson RM, Gibberd RW, Harrison B. Epidemiology of medical error. Br Med J 2000;320:774-7.

7 Ferner RE. Medication errors that have led to manslaughter charges. Br Med J 2000;321:1212-6.

8 Lesar TS, Briceland L, Stein DS. Factors related to errors in medication prescribing. JAMA 1997;277:312-7.

9 Nightingale PG, Adu D, Richards NT, Peters M. Implementation of rules based computerised bedside prescribing and administration: intervention study. Br Med J 2000;320:750-3.

10 Gysling E. Arzneimittelinformation im Internet. pharmakritik 2000;22:61-4.

11 Koller M, Grütter R, Peltenburg M, Fischer JE, Steurer J. Use of the Internet by medical doctors in Switzerland. Swiss Med Wkly 2001;131:251-4.

12 Bates DW. Using information technology to reduce rates of medication errors in hospitals. Br Med J 2000;320:788-91. 